\title{
Uso de beta-bloqueadores en pacientes con enfermedad pulmonar obstructiva crónica
}

\section{Use of beta-blockers in patients with COPD}

José Alex Casallas Osorio(1)

\section{RESUMEN}

La enfermedad pulmonar obstructiva crónica (EPOC) es un síndrome sistémico con múltiples fenotipos, en el que la enfermedad cardiovascular contribuye significativamente a la morbilidad y la mortalidad por su causa. El tabaquismo, la clase socioeconómica baja y un estilo de vida sedentario contribuyen a la historia natural de cada una de estas condiciones. La mayoría de las enfermedades cardiovasculares requieren tratamiento con beta-bloqueadores, que tradicionalmente se han contraindicado en los pacientes con EPOC; sin embargo el uso de estos fármacos se asocia a beneficios y mejores resultados en pacientes con enfermedades cardiovasculares, hipertensión, insuficiencia cardiaca, enfermedad coronaria y enfermedad pulmonar obstructiva crónica.

Palabras clave: receptores adrenérgicos, beta-bloqueadores, enfermedad pulmonar obstructiva crónica, falla cardiaca.

\section{ABSTRACT}

Chronic obstructive pulmonary disease (COPD) is a systemic syndrome with multiple phenotypes. Cardiovascular disease contributes significantly to the morbidity and mortality of COPD. Tobacco use, low socioeconomic class, and sedentary lifestyle contribute to the natural history of each of these conditions. Most cardiovascular diseases require treatment with beta-blockers. Traditionally, beta-blockers have been contraindicated in patients with COPD. However, the use of these drugs is associated with benefits and better results in patients with cardiovascular diseases, hypertension, heart failure, coronary disease, and COPD.

Keywords: adrenergic receptors, beta-blockers, chronic obstructive pulmonary disease, heart failure.

\section{INTRODUCCIÓN}

En la actualidad, la enfermedad pulmonar obstructiva crónica (EPOC) es la cuarta causa de morbilidad y mortalidad en todo el mundo y se espera que en los siguientes años aumente aún más y pase a ser la tercera causa de muerte en el 2020, después de la enfermedad coronaria y el accidente cerebrovascular (1). Es la tercera causa de muerte en los Estados Unidos, con más de 120.000 personas cada año (2). Los estudios en Latinoamérica indican una prevalencia de enfermedad pulmonar obstructiva crónica en individuos mayores de 40 años de 8,9\% a $14,5 \%(3,4)$. En Colombia demanda altos recursos de atención ambulatoria y hospitalaria con alta carga e impacto socioeconómico (5). Es una enfermedad heterogénea y se ha intentado identificar subgrupos (fenotipos)

\footnotetext{
(1) Residente Medicina Interna, Universidad Nacional de Colombia.
}

Correspondencia: José Alex Casallas Osorio. Correo electrónico: jacasallaso@unal.edu.co

Recibido: 15-05-13. Aceptado: 14-06-13. 
que permitan reconocer las diferencias entre individuos con EPOC y enfocar el manejo. Existen tres fenotipos que se asocian con factores pronósticos y diferente respuesta a los tratamientos: agudizador, mixto enfermedad pulmonar obstructiva crónica-asma y enfisema-hiperinsuflado (6-8).

Así, pues, ¿es la enfermedad pulmonar obstructiva crónica un factor de riesgo cardiovascular o es parte del espectro de la enfermedad cardiovascular? La respuesta a esta pregunta se deja a consideración del lector de acuerdo con los aspectos que se plantean a continuación.

En la enfermedad cardiovascular (enfermedad coronaria, enfermedad arterial oclusiva periférica y ataque cerebrovascular) y la enfermedad pulmonar obstructiva crónica existen algunos factores en común:

- Tienen al tabaquismo como factor de riesgo habitual (9), por tanto estos dos trastornos coexisten frecuentemente y pueden ser factores de riesgo interdependientes (10-12). Los otros factores de riesgo son el sedentarismo, el bajo nivel socioeconómico y la mayor incidencia de hipertensión y diabetes en los pacientes con enfermedad pulmonar obstructiva crónica (13).

- Se han propuesto otros factores que enlazan la enfermedad coronaria y la EPOC, como el estado inflamatorio generalizado, el estrés oxidativo, el estrés fisiológico, las alteraciones de la pared vascular, el envejecimiento acelerado y el desequilibrio proteasa/ antiproteasa (13). La elucidación de estos mecanismos puede proporcionar nuevas dianas para el tratamiento.

- Las manifestaciones clínicas de ambas patologías son similares: disnea de esfuerzo, fatiga y reducción en la tolerancia al ejercicio (14).

- La incidencia, morbilidad y mortalidad de ambos aumentan con la edad (1, 15-17). La edad promedio está en crecimiento como resultado de contar con mejores tratamientos para ambas patologías.

- Se sabe que la EPOC es una enfermedad inflamatoria crónica con manifestaciones extrapulmonares significativas (18) y que, además, en la enfermedad cardiovascular la inflamación crónica desempeña un rol importante (19).

- En pacientes con enfermedad pulmonar obstructiva crónica leve a moderada (GOLD 1 y GOLD 2), la principal causa de morbilidad y mortalidad es la enfermedad cardiovascular. Cabe anotar también que en los estadios severos y muy severos (GOLD 3 y GOLD 4) la causa más frecuente de muerte es de origen respiratorio, seguida de la cardiovascular (14).
- En estudios por necropsia en pacientes con enfermedad pulmonar obstructiva crónica que fallecieron en las primeras 24 horas al ingreso, la falla cardiaca representó el $37 \%$ de todas las muertes, seguido por neumonía con $28 \%$ y eventos tromboembólicos con $21 \%$. Sólo el $14 \%$ de las muertes podría atribuirse principalmente a la insuficiencia respiratoria secundaria a la EPOC (20).

- Los pacientes con enfermedad pulmonar obstructiva crónica tienen dos a tres veces más riesgo de hospitalización por afecciones cardiovasculares (incluyendo la enfermedad cardiaca isquémica, ictus e insuficiencia cardíaca) en comparación con aquellos sin EPOC (14).

- La enfermedad coronaria es una indicación absoluta para el uso de beta-bloqueadores $(15,16,21,22)$, pero en el paciente con enfermedad pulmonar obstructiva crónica este tipo de antagonismo se consideraba contraindicado.

- Se ha probado que los beta-bloqueadores no sólo mejoran la morbilidad y mortalidad en enfermedad cardiovascular sino que además lo podrían hacer en la EPOC $(16,23-27)$.

\section{BETA-BLOQUEADORES Y ENFERMEDAD CARDIOVASCULAR}

Los factores de riesgo modificables más importantes en enfermedad cardiovascular son el tabaquismo, la dislipidemia, la diabetes, la hipertensión arterial y la obesidad (29). El número y tipo de fármacos antihipertensivos ha aumentado dramáticamente de 28 en 1972 a más de 125 agentes en la actualidad. Los beta-bloqueadores han estado disponibles para el tratamiento de la hipertensión desde la década de 1960 (tabla 1). Sin embargo, ha habido resistencia al uso de estos fármacos en pacientes con diabetes e insuficiencia renal debido a los efectos secundarios metabólicos. Los betabloqueadores más recientes con efectos vasodilatadores (carvedilol y nebivolol) han demostrado su eficacia y tolerabilidad en pacientes con hipertensión y parecen tener menos efectos adversos. El carvedilol produce vasodilatación por bloqueo alfa y el nebivolol a través de mecanismos mediados por el óxido nítrico (30). La evidencia proveniente de ensayos clínicos hasta la fecha lleva a concluir que los beta-bloqueadores tienen indicación en infarto del miocardio y falla cardiaca con disfunción ventricular izquierda. Sus beneficios incluyen la reducción de la isquemia miocárdica, la reversión de los efectos neurohormonales del sistema nervioso simpático, propiedades antiarrítmicas, efectos sobre la remodelación cardiaca y aumento de la supervivencia $(17,31)$. Existe controversia en cuanto a la selección óptima de un beta-bloqueador, dada la selectividad para los receptores adrenérgicos y sus efectos sobre la circulación periférica. 
Tabla 1. Características farmacológicas de varios beta-bloqueadores (28).

\begin{tabular}{|llllll|}
\hline \multicolumn{1}{|c}{ Fármaco } & $\begin{array}{l}\text { Bloqueo } \\
\text { Selectivo }\end{array}$ & $\begin{array}{c}\text { Simpático- } \\
\text { mimético }\end{array}$ & $\begin{array}{c}\text { Solubilidad } \\
\text { lípidos }\end{array}$ & Eliminación & \multicolumn{1}{c|}{$\begin{array}{c}\text { Cambios } \\
\text { farmacocinéticos }\end{array}$} \\
\hline Acebutolol * & $\beta_{1}$ & $\mathrm{Si}$ & Baja & Renal & $\begin{array}{c}\uparrow \text { concentración plasma } \\
\text { (t } 1 / 2)\end{array}$ \\
Atenolol * & $\beta_{1}$ & $\mathrm{No}$ & Baja & Renal & $\downarrow$ depuración, $\uparrow \mathrm{t} 1 / 2$ \\
Bisoprolol * & $\beta_{1}$ & $\mathrm{No}$ & $\mathrm{Baja}$ & Renal/hepática & No cambios \\
Metoprolol * & $\beta_{1}$ & $\mathrm{No}$ & Moderada & Hepática & No cambios \\
Nebivolol * & $\beta_{1}$ & $\mathrm{No}$ & Baja & Renal/hepática & $\downarrow$ depuración \\
Carvedilol & $\beta_{1}+\beta_{2}, \alpha_{1}$ & $\mathrm{No}$ & Moderada & Hepática & $\uparrow \mathrm{AUC}, \downarrow$ depuración \\
Labetalol & $\beta_{1}+\beta_{2}, \alpha_{1}$ & $\mathrm{Si}($ débil) & Moderada & Hepática & $\downarrow$ depuración, $\uparrow \mathrm{t} 1 / 2$ \\
Propranolol & $\beta_{1}+\beta_{2}$ & $\mathrm{No}$ & Alta & Hepática & $\uparrow$ concentración plasma \\
Sotalol & $\beta_{1}+\beta_{2}$ & $\mathrm{No}$ & Baja & Renal & $\uparrow$ concentración plasma \\
Nadolol & $\beta_{1}+\beta_{2}$ & $\mathrm{No}$ & Baja & Renal & $\uparrow \mathrm{t} 1 / 2$ \\
Pindolol & $\beta_{1}+\beta_{2}$ & $\mathrm{Si}$ & Moderada & Renal & $\uparrow \mathrm{AUC}, \uparrow \mathrm{t}_{1 / 2}$ \\
\hline
\end{tabular}

${ }^{*}$ Beta-bloqueador cardioselectivo.

t 1/2: vida media

AUC: área bajo la curva

Varios estudios disponibles tienen muestras pequeñas y corta duración del seguimiento, lo cual limita sus conclusiones. Otros pocos han tratado de responder algunas preguntas cruciales como la tolerabilidad a los beta-bloqueadores en la insuficiencia cardíaca y la superioridad de un beta-bloqueador dado sobre otro en la prevención de eventos relevantes. Solo un estudio sugiere que el carvedilol aporta un mayor beneficio en supervivencia que el metoprolol en pacientes con insuficiencia cardíaca (NYHA II-IV) tratados con diuréticos e inhibidores de la enzima conversora de angiotensina (IECA). El carvedilol incrementa la supervivencia una mediana de 1,4 años (IC95\%: 0,5-2,3) respecto al metoprolol. Este resultado favorable para carvedilol puede atribuirse al bloqueo de los receptores beta 1 , beta 2 y alfa-adrenérgicos, al mayor efecto anti-isquémico, a la inhibición de la apoptosis, a la acción antioxidante, a la eliminación de radicales libres o a un efecto electrofisiológico (32). Otra situación de largo debate, en este caso en enfermedad coronaria, es que los beneficios observados en los ensayos clínicos no concuerdan o no se evidencian en grandes estudios observacionales (21).

\section{BETA-BLOQUEADORES Y ENFERMEDAD PULMONAR OBSTRUCTIVA CRÓNICA}

Ya en 1980 se cuestionaba la conveniencia o no del uso de beta-bloqueadores en el tratamiento de las enfermedades cardiovasculares en los pacientes con EPOC, y se identificaba la coexistencia de entre ambas enfermedades (33). En un estudio de ese entonces, en un grupo de pacientes con enfermedad pulmonar obstructiva crónica y enfermedad coronaria o hipertensión, se observó un incremento en cuanto a obstrucción con el uso del metoprolol frente a placebo, pero en menor proporción que con el propranolol (34). Por esta razón, en la EPOC han sido subutilizados debido al temor de broncoconstricción y efectos adversos, sobre todo en las personas mayores. Los pacientes con enfermedad pulmonar obstructiva crónica corresponden a edades mayores, y tienen más comorbilidades así como menor uso de beta-bloqueadores en comparación con aquellos sin ésta $(17,35)$.

La información disponible en la actualidad indica que los pacientes con EPOC toleran bien los beta-bloqueadores cardioselectivos y que tienen un beneficio en morbilidad y mortalidad a largo plazo. Los beta-bloqueadores reducen la mortalidad en los pacientes con enfermedad pulmonar obstructiva crónica y enfermedad cardiovascular y deben utilizarse siempre que sea posible. Si existe indicación, los beta-bloqueadores cardioselectivos son seguros en pacientes con EPOC, de ahí que se pueda iniciarlos y titular la respuesta dado el beneficio demostrado en enfermedades como la insuficiencia cardiaca, la enfermedad coronaria y la hipertensión arterial (15-17, 22-27, 36-43). 


\section{CONCLUSIONES}

La tabla 2 resume las principales conclusiones que se pueden derivar de la evidencia de la literatura médica con relación al empleo de beta-bloqueadores en
EPOC, su suspensión en el paciente estable así como durante la exacerbación, el empleo de beta-bloqueadores cardioselectivos, el tratamiento de la hipertensiòn pulmonar en el paciente con EPOC y el empleo de beta-bloqueadores en el fenotipo asma/EPOC.

\section{Tabla 2.}

\begin{tabular}{|c|c|c|}
\hline Tema & Conclusión & Referencias \\
\hline \multirow[b]{2}{*}{ Uso beta-bloqueadores en EPOC } & $\begin{array}{l}\text { Los pacientes con EPOC tienen edades mayores y más comorbilida- } \\
\text { des cardiovasculares y a pesar de ello tienen menor prescripción de } \\
\text { beta-bloqueadores en comparación con aquellos sin EPOC }\end{array}$ & 17,35 \\
\hline & $\begin{array}{l}\text { En un estudio prospectivo recientemente publicado se encontró, en el } \\
\text { grupo de pacientes con EPOC severa oxígeno-dependientes, un incre- } \\
\text { mento en la mortalidad con el uso de beta-bloqueadores (IC } 95 \% \text { 1,04- } \\
1,37 ; p=0,010) \text {. No obstante, varios estudios retrospectivos previos han } \\
\text { mostrado un efecto benéfico de los beta-bloqueadores con relación a } \\
\text { supervivencia y exacerbaciones en el paciente con EPOC. }\end{array}$ & $23-27,44$ \\
\hline $\begin{array}{l}\text { Suspensión de beta-bloqueadores } \\
\text { en EPOC estable }\end{array}$ & $\begin{array}{l}\text { La evidencia acumulada de estudios y meta-análisis indica que no de- } \\
\text { ben suspenderse los beta-bloqueadores en los pacientes con EPOC }\end{array}$ & 23,39 \\
\hline $\begin{array}{l}\text { Suspensión de beta-bloqueadores } \\
\text { en la exacerbación de EPOC }\end{array}$ & $\begin{array}{l}\text { No tiene ningún beneficio retirar el beta bloqueador, luego deben con- } \\
\text { tinuar con el tratamiento }\end{array}$ & $25,26,41,42$ \\
\hline Paciente anciano & $\begin{array}{l}\text { Los beta-bloqueadores cardioselectivos parecen ser seguros para su } \\
\text { uso en pacientes ancianos con insuficiencia renal leve a moderada y } \\
\text { EPOC, que tienen una indicación absoluta para la terapia con beta- } \\
\text { bloqueador. }\end{array}$ & 15,45 \\
\hline \multirow{2}{*}{$\begin{array}{l}\text { Beta-bloqueadores } \\
\text { cardioselectivos vs. no } \\
\text { cardioselectivos }\end{array}$} & $\begin{array}{l}\text { En principio se debe preferir el uso de un beta-bloqueador cardiose- } \\
\text { lectivo, excepto en pacientes con insuficiencia cardíaca que podrían } \\
\text { beneficiarse de manera significativa del uso de carvedilol. }\end{array}$ & $\begin{array}{l}15-17,23,24 \\
39,40\end{array}$ \\
\hline & $\begin{array}{l}\text { En pacientes con EPOC y enfermedad coronaria o cardiovascular que } \\
\text { requieren beta-bloqueador es preferible usar uno cardioselectivo tipo } \\
\text { bisoprolol, metoprolol succinato, nevibolol y evitar aquellos que no per- } \\
\text { tenezcan a este grupo. }\end{array}$ & $16,22,23,30$ \\
\hline $\begin{array}{l}\text { Cambio de beta-bloqueador } \\
\text { cardioselectivo a no selectivo }\end{array}$ & $\begin{array}{l}\text { El carvedilol, el metoprolol succinato y el bisoprolol son los beta-blo- } \\
\text { queadores en el tratamiento de falla cardiaca y no está caracterizado } \\
\text { por completo si las diferencias en las especificidades del receptor beta } \\
\text { afectan la función pulmonar o vascular en pacientes con falla cardiaca, } \\
\text { particularmente en aquellos con EPOC coexistente. El cambio entre } \\
\text { beta-bloqueador selectivo y no selectivo se tolera bien, pero hay alte- } \\
\text { raciones leves en las pruebas de función respiratoria pulmonar, más } \\
\text { marcada en los pacientes con EPOC }\end{array}$ & 46 \\
\hline $\begin{array}{l}\text { Tratamiento de la hipertensión en } \\
\text { el paciente con EPOC }\end{array}$ & $\begin{array}{l}\text { En los pacientes con EPOC e hipertensión que requieran más de dos } \\
\text { agentes antihipertensivos, la terapia de combinación incluyendo un diu- } \\
\text { rético tiazídico, se asoció con un riesgo significativamente menor de } \\
\text { hospitalizaciones por falla cardiaca en pacientes sin diagnóstico pre- } \\
\text { vio de insuficiencia cardíaca congestiva. Las diferentes combinaciones } \\
\text { antihipertensivas no tuvieron una asociación significativa con el riesgo } \\
\text { de exacerbación de la enfermedad pulmonar obstructiva crónica ((in- } \\
\text { hibidores de la enzima convertidora de angiotensina o de su receptor, } \\
\text { calcioantagonistas) }\end{array}$ & 47 \\
\hline $\begin{array}{l}\text { Beta-bloqueadores en asma y en } \\
\text { fenotipo mixto asma/EPOC }\end{array}$ & $\begin{array}{l}\text { En los pacientes con asma los beta-bloqueadores pueden llevar a em- } \\
\text { peoramiento o agudización de los síntomas. Esto es relevante en el } \\
\text { fenotipo mixto del paciente con EPOC; estos pacientes en conjunto } \\
\text { representan entre el } 20 \% \text { y el } 40 \% \text { de todos los pacientes con EPOC }\end{array}$ & $6-8$ \\
\hline
\end{tabular}




\section{BIBLIOGRAFÍA}

1. Global Initiative for chronic obstructive lung disease GOLD. Global Strategy for the Diagnosis, Management and Prevention of COPD. 2013. Disponible en: http://www.goldcopd.org.

2. Minino AM, Murphy SL, Xu J, Kochanek KD. Deaths: final data for 2008. National vital statistics reports : from the Centers for Disease Control and Prevention, National Center for Health Statistics, National Vital Statistics System. 2011; 59: 1-126. PubMed PMID: 22808755

3. Menezes AM, Pérez-Padilla R, Jardim JR, Muino A, López MV, Valdivia $G$, et al. Chronic obstructive pulmonary disease in five Latin American cities (the PLATINO study): a prevalence study. Lancet. 2005; 366 (9500): 1875-81.

4. Caballero As, Torres-Duque CA, Jaramillo C, Bolívar F, Sanabria $F$, Osorio P, et al. Prevalence of COPD in Five Colombian Cities Situated at Low, Medium, and High Altitude (PREPOCOL Study)*. CHEST Journal. 2008; 133: 343-9.

5. Ministerio de Salud y Protección Social. Tomo III-Morbilidad y mortalidad de la población colombiana. 2010. Disponible en: http://www.minsalud.gov.co/salud/Paginas/An\%C3\%A1lisis-dela-Situaci\%C3\%B3n-de-Salud-en-Colombia.aspx.

6. Miravitlles M, Calle M, Soler-Cataluña JJ. Clinical phenotypes of COPD: identification, definition and implications for guidelines. Archivos de Bronconeumología. 2012; 48: 86-98.

7. Louie S, Zeki AA, Schivo M, Chan AL, Yoneda KY, Avdalovic M, et al. The asthma-chronic obstructive pulmonary disease overlap syndrome: pharmacotherapeutic considerations. Expert review of clinical pharmacology. 2013; 6: 197-219.

8. Carolan BJ, Sutherland ER. Clinical phenotypes of chronic obstructive pulmonary disease and asthma: Recent advances. J Allerg Clin Immunol. 2013; 131: 627-34.

9. Centers for Disease Control and Prevention (CDC). Smokingattributable mortality, years of potential life lost, and productivity losses-United States, 2000-2004. MMWR Morbidity and mortality weekly report. 2008; $57: 1226-8$.

10. Man JP, Sin DD, Ignaszewski A, Man SF. The complex relationship between ischemic heart disease and COPD exacerbations. Chest. 2012; 141: 837-8.

11. Patel ARC, Donaldson GC, Mackay AJ, Wedzicha JA, Hurst JR. The Impact of ischemic heart disease on symptoms, health status, and exacerbations in patients with COPD impact of ischemic heart disease on COPD. CHEST Journal. 2012; 141: 851-7.

12. Chatterjee S. A 13-item score based on readily available risk factors predicted risk for 3-year mortality in heart failure. Ann Intern Med. 2013; 158 (2).

13. Maclay JD, MacNee W. Cardiovascular disease in COPD: Mechanisms. CHEST Journal. 2013; 143: 798-807.

14. Sin DD. Is COPD really a cardiovascular disease? CHEST Journal. 2009; 136: 329-30.

15. Andrus MR, Loyed JV. Use of beta-adrenoceptor antagonists in older patients with chronic obstructive pulmonary disease and cardiovascular co-morbidity: safety issues. Drugs \& Aging. 2008; 25: 131-44.

16. Albouaini K, Andron M, Alahmar A, Egred M. Beta-blockers use in patients with chronic obstructive pulmonary disease and concomitant cardiovascular conditions. Int J Chronic Obstructive Pulmonary Disease. 2007; 2: 535-40.

17. Mentz R, Wojdyla D, Fiuzat M, Chiswell K, Fonarow G, O'Connor C. Association of beta-blocker use and selectivity with outcomes in patients with heart failure and chronic obstructive pulmonary disease (from OPTIMIZE-HF). Am J Cardiol. 2013; 111: 582-7.

18. Nussbaumer-Ochsner Y, Rabe KF. Systemic Manifestations of COPD. CHEST Journal. 2011; 139: 165-73.

19. Ertek S, Cicero A. Impact of physical activity on inflammation: effects on cardiovascular disease risk and other inflammatory conditions. Archives of medical science: AMS. 2012; 8: 794-804.

20. Zvezdin B, Milutinov S, Kojicic M, Hadnadjev M, Hromis S, Markovic M, et al. A Postmortem analysis of major causes of early death in patients hospitalized with COPD Exacerbation. CHEST Journal. 2009; 136: 376-80.

21. Bangalore S, Steg G, Deedwania P, Crowley K, Eagle KA, Goto $\mathrm{S}$, et al. beta-Blocker use and clinical outcomes in stable outpatients with and without coronary artery disease. JAMA. 2012; 308: $1340-9$.

22. Angeloni E, Melina G, Roscitano A, Refice S, Capuano F, Lechiancole $A$, et al. $\beta$-Blockers improve survival of patients with chronic obstructive pulmonary disease after coronary artery bypass grafting. Ann Thorac Surg. 2013; 95: 525-31.

23. Salpeter S, Ormiston T, Salpeter E. Cardioselective beta-blockers for chronic obstructive pulmonary disease. Cochrane database of systematic reviews (Online). 2005: CD003566.

24. van Gestel YRBM, Hoeks SE, Sin DD, Welten GMJM, Schouten $\mathrm{O}$, Witteveen HJ, et al. Impact of cardioselective $\beta$-blockers on mortality in patients with chronic obstructive pulmonary disease and atherosclerosis. Am J Resp Crit Care Med. 2008; 178: 695700.

25. Rutten Fh ZNAHEGDEHAW. B-blockers may reduce mortality and risk of exacerbations in patients with chronic obstructive pulmonary disease. Arch Int Med. 2010; 170: 880-7.

26. Zeng Q, Jiang S. Update in diagnosis and therapy of coexistent chronic obstructive pulmonary disease and chronic heart failure. J Thorac Dis. 2012; 4: 310-5.

27. Etminan M, Jafari S, Carleton B, FitzGerald J. Beta-blocker use and COPD mortality: a systematic review and meta-analysis. BMC Pulmonary Medicine. 2012; 12: 48.

28. Brunton L, Chabner B, Knollman B. Goodman and Gilman's The Pharmacological Basis of Therapeutics, Twelfth Edition: McGraw-Hill Education; 2010.

29. Laslett LJ, Alagona P, Jr., Clark BA, Drozda JP, Jr., Saldivar F, Wilson SR, et al. The worldwide environment of cardiovascular disease: prevalence, diagnosis, therapy, and policy issues: a report from the American College of Cardiology. J Am Coll Cardiol. 2012; 60: S1-49.

30. Black HR, Greenberg BH, Weber MA. The foundation role of beta blockers across the cardiovascular disease spectrum: a year 2009 update. Am J Med. 2010; 123: S2.

31. Saurav C, Giuseppe B-Z, Antonio A, Fabrizio DA, Davide C, Benjamin Van $T$, et al. Benefits of $\beta$ blockers in patients with heart failure and reduced ejection fraction: network meta-analysis. BMJ. 2013; 346.

32. Poole-Wilson PA, Swedberg K, Cleland JGF, Di Lenarda A, Hanrath $\mathrm{P}$, Komajda $\mathrm{M}$, et al. Comparison of carvedilol and metoprolol on clinical outcomes in patients with chronic heart failure in the Carvedilol Or Metoprolol European Trial (COMET): randomised controlled trial. The Lancet. 2003; 362: 7-13.

33. John W. Jenne MD. Beta blockers and COPD. Chest. 1980; 78: 675-6. 
34. Wunderlich J, Macha HN, Wudicke H, Huckauf H. Beta-adrenoceptor blockers and terbutaline in patients with chronic obstructive lung disease. Effects and interaction after oral administration. CHEST Journal. 1980; 78: 714-20.

35. Ege M, Guray U, Guray Y, Yilmaz M, Yucel O, Zorlu A, et al. Acute heart failure with accompanying chronic obstructive pulmonary disease: should we focus on beta blockers? Herz. 2012; 37: 796-800.

36. Short PM, Lipworth SIW, Elder DHJ, Schembri S, Lipworth BJ. Effect of $\beta$ blockers in treatment of chronic obstructive pulmonary disease: a retrospective cohort study. BMJ. 2011; 342.

37. Bryant L. $\beta$-blockers in COPD - yes, it's OK (with care). J Primary Health Care. 2012; 4: 165.

38. Suissa S, Ernst P. $\beta$-blockers for COPD inpatients. Thorax. 2012; 67: 936-7.

39. Ni Y, Shi G, Wan H. Use of cardioselective $\beta$-blockers in patients with chronic obstructive pulmonary disease: a meta-analysis of randomized, placebo-controlled, blinded trials. J Int Med Research. 2012; 40: 2051-65.

40. Mainguy V, Girard D, Maltais F, Saey D, Milot J, Sénéchal M, et al. Effect of bisoprolol on respiratory function and exercise capacity in chronic obstructive pulmonary disease. Am J Cardiol. 2012; 110: 258-63.

41. Stefan $M$, Rothberg M, Priya A, Pekow P, Au D, Lindenauer P. Association between $\beta$-blocker therapy and outcomes in patients hospitalised with acute exacerbations of chronic obstructive lung disease with underlying ischaemic heart disease, heart failure or hypertension. Thorax. 2012; 67: 977-84.
42. Stefan MS, Priya A, Rothberg M, Pekow PS, Lindenauer P. The effectiveness and safety of beta-blocker therapy for patients hospitalized for acute exacerbations of chronic obstructive lung disease with underlying comorbid ischemic heart disease or heart failure. Am J Respiratory Crit Care Med. 2012; 185 (1 MeetingAbstracts): A6638.

43. Mentz R, Schulte P, Fleg J, Fiuzat M, Kraus W, Piña I, et al. Clinical characteristics, response to exercise training, and outcomes in patients with heart failure and chronic obstructive pulmonary disease: Findings from Heart Failure and A Controlled Trial Investigating Outcomes of Exercise TraiNing (HF-ACTION). Am Heart J. 2013; 165: 193-9.

44. Ekström M, Bornefalk Hermansson A, Ström K. Effects of cardiovascular drugs on mortality in severe COPD: a time-dependent analysis. Am J Respiratory Crit Care Med. 2013;187: 715-20.

45. Lainscak M, Gelbrich G, Inkrot S, Tahirovic E, Edelmann F, Doehner W, et al. Pulmonary function during beta-blocker titration in elderly patients with heart failure: The CIBIS-ELD Analysis. Am J Respiratory Crit Care Med. 2010; 181 (1 MeetingAbstracts): A6850.

46. Jabbour A, Macdonald PS, Keogh AM, Kotlyar E, Mellemkjaer $S$, Coleman CF, et al. Differences between beta-blockers in patients with chronic heart failure and chronic obstructive pulmonary disease: a randomized crossover trial. J Am Coll Cardiol. 2010; 55: 1780-7.

47. Herrin M, Feemster L, Crothers K, Uman J, Bryson C, Au D. Combination antihypertensive therapy among patients with COPD. Chest. 2013;143: 1312-20. 